\title{
A catalogue of quasars and active nuclei: 12th edition ${ }^{\star}$
}

\author{
M.-P. Véron-Cetty and P. Véron
}

Observatoire de Haute Provence, CNRS, 04870 Saint-Michel l'Observatoire, France

e-mail: [mira.veron;philippe.veron]@oamp . fr

Received 9 March 2006 / Accepted 13 April 2006

\section{ABSTRACT}

\begin{abstract}
Aims. This catalogue is aimed at presenting a compilation of all known AGN in a compact and convenient form and we hope that it will be useful to all workers in this field.

Methods. Like the eleventh edition, it includes position and redshift as well as photometry $(U, B, V)$ and $6 \mathrm{~cm}$ flux densities when available. We now give $20 \mathrm{~cm}$ rather than $11 \mathrm{~cm}$ flux densities.

Results. The present version contains 85221 quasars, 1122 BL Lac objects and 21737 active galaxies (including 9628 Seyfert 1s), almost doubling the number listed in the 11th edition. We also give a list of all known lensed and double quasars.
\end{abstract}

Key words. galaxies: quasars: general - galaxies: Seyfert - galaxies: BL Lacertae objects: general

\section{Introduction}

The first catalogue of quasars was published in 1971 by De Veny et al. It contained 202 objects. The number of known quasars has since steadily increased until the year 2000 (see Table 1). The release of both the $2 \mathrm{dF}$ catalogue (Croom et al. 2001, 2003) and the first part (Abazajian et al. 2003) of the "Sloan Digital Sky Survey" (Fan et al. 1999) has dramatically increased the number of known quasars justifying the 10th and 11th editions of the present catalogue. The recent release of the last three installments of the SDSS (Abazajian et al. 2004, 2005; Adelman-McCarthy et al. 2006) which has again almost doubled the number of known quasars, made a new edition timely.

This edition contains quasars with measured redshift known to us prior to January 1st, 2006; as in the preceding editions, we do not give any information about absorption lines or X-ray properties. But we give the absolute magnitude ${ }^{1}$ for each object and, when available, 20 and $6 \mathrm{~cm}$ flux densities.

This catalogue should not be used for any statistical analysis as it is not complete in any sense, except that it is, we hope, a complete survey of the literature.

\section{Description of the catalogue}

We have arbitrarily defined a quasar as a starlike object, or an object with a starlike nucleus, with broad emission lines and brighter than absolute magnitude $M_{B}=-23$. The quasars are listed in Table_QSO. A sample page is shown in Fig. 1. Clearly, some objects would move from Table_QSO to Table_AGN and vice versa if other values for $q_{0}$ and the spectral index were used or if an accurate B apparent magnitude was available for all objects. The variability may have a similar effect, as well as the size of the diaphragm used for the measurement as the contribution of the underlying galaxy for low- $z$ quasars may not be negligible.

* The catalogue (Table_QSO, Table_BL, Table_AGN and Table_reject) and the list of references are only available in electronic form at CDS via anonymous ftp to

cdsarc.u-strasbg.fr $(130.79 .128 .5)$ or via

http://cdsweb.u-strasbg.fr/cgi-bin/qcat?J/A+A/455/773 or at the Observatoire de Haute Provence (http://www . obs-hp.fr/)

${ }^{1} H_{0}=50 \mathrm{~km} \mathrm{~s}^{-1} \mathrm{Mpc}^{-1}$ and $q_{0}=0$ is assumed throughout this paper.
Table 1. Increase with time of the number of known QSOs, BL Lacs and Seyfert 1s.

\begin{tabular}{rrrl}
\hline \hline QSO & BL Lac & Seyfert 1 & reference \\
\hline 202 & & & De Veny et al. (1971) \\
2251 & & 190 & Véron-Cetty \& Véron (1984) \\
2835 & 73 & 236 & Véron-Cetty \& Véron (1985) \\
3473 & 84 & 258 & Véron-Cetty \& Véron (1987) \\
4169 & 117 & 358 & Véron-Cetty \& Véron (1989) \\
6225 & 162 & 575 & Véron-Cetty \& Véron (1991) \\
7383 & 171 & 695 & Véron-Cetty \& Véron (1993) \\
8609 & 220 & 888 & Véron-Cetty \& Véron (1996a) \\
11358 & 357 & 1111 & Véron-Cetty \& Véron (1998) \\
13214 & 462 & 1711 & Véron-Cetty \& Véron (2000a) \\
23760 & 608 & 2765 & Véron-Cetty \& Véron (2001) \\
48921 & 876 & 11777 & Véron-Cetty \& Véron (2003) \\
85221 & 1122 & 9628 & Present edition \\
\hline
\end{tabular}

In Table_BL, we list all confirmed, probable or possible BL Lac objects with or without a measured redshift, without consideration of their absolute magnitude. As better spectra are becoming available, broad emission lines have been detected in a number of objects formerly classified as BL Lac; they have usually been moved to Table QSO (Véron-Cetty \& Véron 2000b).

Table_AGN lists "active galaxies": Seyfert 1s, Seyfert 2 s and Liners fainter than $M_{B}=-23^{2}$. A number of galaxies with a nuclear H II region are also included (158), the reason being that they have been called AGN in the past and later reclassified; we consider it useful to keep track of these reclassifications to avoid further confusion.

Seyfert 1s have broad Balmer and other permitted lines; Seyfert 2s have Balmer and forbidden lines of the same width. Osterbrock $(1977,1981)$ has divided the Seyfert 1s into five subgroups: Seyfert 1.0, 1.2, 1.5, 1.8 and 1.9 on the basis of the appearance of the Balmer lines. Seyfert 1.0s are "typical" members of the class, as described by Khachikian \& Weedman (1971, 1974), while Seyfert 1.5s are objects intermediate between typical Seyfert $1 \mathrm{~s}$ and Seyfert $2 \mathrm{~s}$, with an easily apparent

\footnotetext{
${ }^{2}$ In the 11th edition, we included 5015 galaxies from the SDSS first release, classified as QSOs on the basis of their colours only. We thank Jenny Greene who has pointed out this error which is now corrected.
} 

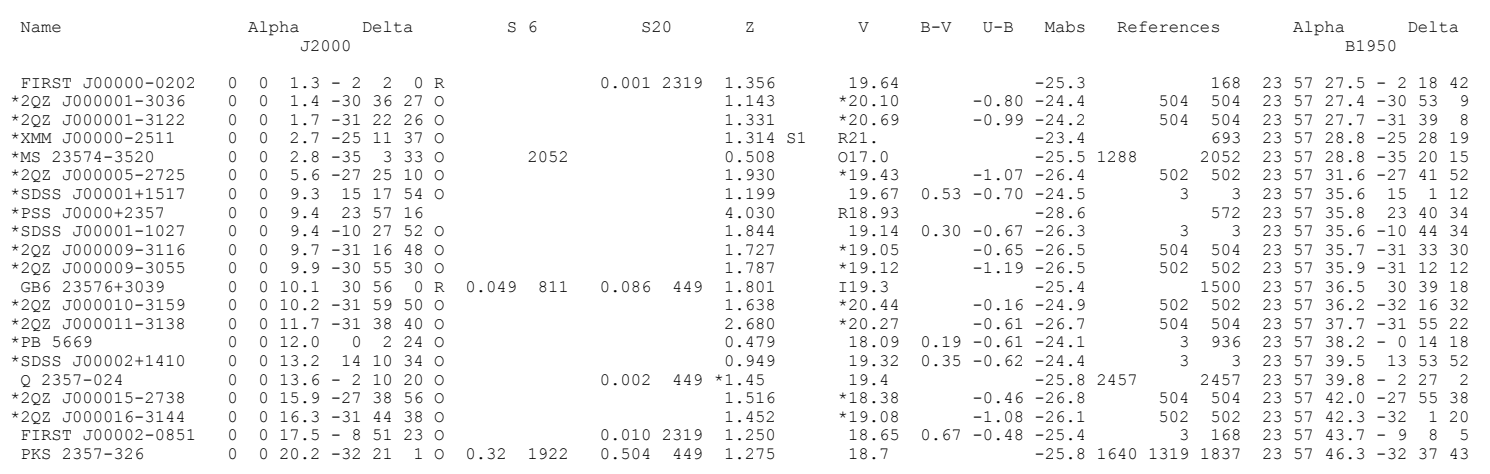

Fig. 1. Sample page of the QSO catalogue.

narrow $\mathrm{H} \beta$ profile superimposed on broad wings. The classes Seyfert 1.2 and 1.8 are used to describe objects with relatively weaker and stronger narrow $\mathrm{H} \beta$ components, intermediate between Seyfert 1.0 and 1.5 and Seyfert 1.5 and 2 respectively. In Seyfert 1.9, although the broad $\mathrm{H} \alpha$ emission is clearly seen, broad $\mathrm{H} \beta$ cannot be detected with certainty by mere visual inspection of the spectra. We have adopted the more quantitative classification introduced by Winkler (1992):

$\begin{array}{rl}\mathrm{S} 1.0 & 5.0<R \\ \mathrm{~S} 1.2 & 2.0<R<5.0 \\ \mathrm{~S} 1.5 & 0.33<R<2.0 \\ \mathrm{~S} 1.8 & \quad R<0.33\end{array}$

S1.9

$\mathrm{S} 2$

$\mathrm{Q} 2$

where $R$ is the ratio of the total $\mathrm{H} \beta$ to the [OIII] $\lambda 5007$ fluxes. Several objects have been found to show extreme spectral variability, changing from Seyfert 1.8 or 1.9 to Seyfert 1.0. In some cases these changes are consistent with changes of the reddening towards the BLR while, in others, they are probably due to real changes in ionizing flux (Goodrich 1989a, 1995; Tran et al. 1992b). In some Seyfert 2s, a broad $\mathrm{Pa} \beta$ line has been detected, indicating the presence of a highly reddened broad line region (Goodrich et al. 1994); we call these objects S1i. A number of Seyfert $2 \mathrm{~s}$ have, in polarized light, the spectra of Seyfert 1s (Antonucci \& Miller 1985; Miller \& Goodrich 1990; Tran et al. 1992a); we call them S1h. Typical full widths at half-maximum of the Balmer lines in Seyfert 1s lie in the range $2000-6000 \mathrm{~km} \mathrm{~s}^{-1}$; however, there is a group of active galactic nuclei with all the properties of Seyfert 1s, but with unusually narrow Balmer lines (Osterbrock \& Pogge 1985; Goodrich 1989b); they are defined as having the broad component of the Balmer lines narrower than $2000 \mathrm{~km} \mathrm{~s}^{-1}$ FWHM (Osterbrock 1987); we call them S1n. Liners (as defined by Heckman 1980) are called S3. If broad Balmer lines are observed, they are called S3b; if these broad Balmer lines are only seen in polarized light, they are called S3h.

Seyfert 1 galaxies and QSOs, when viewed through the absorbing dusty torus have the same optical appearance; however, they differ either by their hard X-ray luminosity or, for radio loud objects, by their radio luminosity. It has become customary to call type 2 QSOs (or Q2) rather than Seyfert 2 the high luminosity narrow line objects. Treister et al. (2005) call type 2 QSOs narrow line objects with $L_{0.5-10 \mathrm{keV}}>10^{42} \mathrm{erg} \mathrm{s}^{-1}\left(H_{0}=\right.$ $70 \mathrm{~km} \mathrm{~s}^{-1} \mathrm{Mpc}^{-1}$ ) or $>10^{42.3} \mathrm{erg} \mathrm{s}^{-1}$ if $H_{0}=50 \mathrm{~km} \mathrm{~s}^{-1} \mathrm{Mpc}^{-1}$, while Derry et al. (2003) have more conservatively defined
QSO2s as having an intrinsic, hard (2-10 keV) X-ray luminosity larger than $10^{44.3} \mathrm{erg} \mathrm{s}^{-1}$. Véron-Cetty \& Véron (2000b) have shown that narrow line objects with a $178 \mathrm{MHz}$ radio luminosity $S_{178}>10^{36} \mathrm{erg} \mathrm{s}^{-1} \mathrm{~Hz}^{-1}$ are QSO2s rather than Seyfert $2 \mathrm{~s}$.

In Table_AGN, 6875 objects have no classification. Most of them were originally classified as QSOs but turned out to be fainter than $M_{B}=-23$ and were therefore moved to this table.

Table_reject lists the objects which once were believed to be AGN and are now known to be either stars or normal galaxies.

Table_QSO contains 85221 objects, Table_BL, 1122, Table_AGN, 21737 and Table_reject, 141. The catalogue is believed to contain all known quasars, BL Lac objects and Seyfert 1s.

Table_QSO, Table_BL and Table_AGN give:

1) Columns 1 and 2 . The most common name of the object. For the meaning and the sources of the designations see Hewitt \& Burbidge (1987), Fernandez et al. (1983) and Kesteven $\&$ Bridle (1977). For the sources discovered by the ROSAT X-ray satellite, we have used the following acronyms: RXS for the sources appearing in the All-Sky Bright Source Catalogue (Voges et al. 1999), 1WGA for the sources published in the WGACAT catalogue (White et al. 1994) and RX for the others.

When the name is preceded by an *, the object has not been explicitly associated with a radio source.

2) Columns 3 to 10 . The best available J2000 optical or radio coordinates. The J2000 positions have been converted from the B1950 positions using the matrix given by Aoki et al. (1983). An $O$ or an $R$ following the coordinates means that the position is either an optical or a radio position measured with an accuracy better than one arcsec. An $A$ means that it is only an approximate position which may be wrong by several arc minutes. No reference is given for the source of the positions. The availability of the Digitized Sky Survey (DSS) allows quick measurements of the optical position of any object brighter than $\approx 19.5$ mag. It has already been used to measure the position of several hundreds QSOs (Schneider et al. 1992; Bowen et al. 1994; Kirhakos et al. 1994; Véron-Cetty \& Véron 1996b). Optical positions with an accuracy better than $2^{\prime \prime}$ have also been measured for the 19369 galaxies in the Zwicky catalogue (Falco et al. 1999) and for the 12921 UGC galaxies (Cotton et al. 1999).

3) Columns 11 to 14. The 6 and $20 \mathrm{~cm}$ flux densities (in Jy) with references to the literature. When several measurements are available we took arbitrarily one of them. When a reference is given for the $6 \mathrm{~cm}$ flux density but the value of the flux density itself is left blank and there is an * in Col. 1, only an upper limit is available and this upper limit is not much greater than $1 \mathrm{mJy}$; in case there is no $*$ in Col. 1 , the reference refers to a detection but at a wavelength other than $6 \mathrm{~cm}$.

The $20 \mathrm{~cm}$ flux densities have been taken mainly from the NRAO VLA Sky Survey (NVSS) (Condon et al. 1998) and the 
FIRST survey (Becker et al. 1995; White et al. 1997). The NVSS covers the sky north of $\delta(\mathrm{J} 2000.0)=-40^{\circ}$. The catalog contains 1814748 discrete sources stronger than $S \sim 2.5 \mathrm{mJy}$. The resolution was $45^{\prime \prime} F W H M$. The rms uncertainties in $\alpha$ and $\delta$ vary from $\leq 1^{\prime \prime}$ for the sources stronger than $15 \mathrm{mJy}$ to $7^{\prime \prime}$ at the survey limit. The FIRST survey was carried out with the VLA. It covers an area of $9033 \mathrm{deg}^{2}$ to a sensitivity limit of $\sim 1 \mathrm{mJy}$. The catalog contains 811118 sources. Source positions are good to better than $1^{\prime \prime}$. The beam size was 5 .'4. Identifications of FIRST radio sources with the 2001 version of the present catalogue were previously attempted by Wadadekar (2004) who found 775 coincidences.

4) Columns 15 and 16. The redshift as published. An* in front of the redshift means that it has been estimated from a low dispersion slitless spectrum and is of lesser accuracy or even plainly wrong as the emission lines may easily be misidentified. We have given only those values which are described as probable in the original sources and not the possible values.

5) Column 17. In this column an attempt has been made to classify the objects as S1, S1.0, S1.2, S1.5, S1.8, S1.9, S1i, S1h, S1n, S2, Q2, S3, S3b, S3h, S, S? or H2. Low redshift quasars are classified as $\mathrm{S} 1$ when a good spectrum shows that they are similar to Seyfert 1 galaxies.

In Table_BL, we find in this column:

$\begin{array}{ll}\text { BL } & \text { for a confirmed BL Lac object. } \\ \text { BL? } & \text { for a probable BL Lac } \\ \text { blank } & \text { for a possible BL Lac. } \\ ? & \text { for a questionable BL Lac } \\ \text { HP } & \text { for a Highly Polarized object. }\end{array}$

6) Columns 18 to 21 . The $V, B-V$ and $U-B$ photoelectric or photographic magnitude and colours, when available (the survey of the literature for photographic colours may be incomplete) (an * in front of the magnitude indicates that the colours and the magnitude are photographic, while an $R$ or an $I$ indicates a red or an infrared magnitude). The column labelled " $V$ " gives the $V$ magnitude when $B-V$ is also given. When $B-V$ is not given, this column usually gives the $B$ magnitude, unless it is preceded by an $R$ or an $I$. Maoz et al. (1993) have measured homogeneous $V$ magnitudes for 354 QSOs with an accuracy of $\pm 0.1 \mathrm{mag}$; they have been included. For a few objects the $O$ magnitude, measured on the blue Palomar Sky Survey plates, or the UK Science Research Council SRC-J Survey plates, believed to be accurate within $\pm 0.2 \mathrm{mag}$, has been extracted from the APS database (Pennington et al. 1993). For a number of objects we give the $O$ magnitude, extracted from the USNO-A2 catalogue (Monet et al. 1996) or the Cambridge Automated Plate Measuring Machine (APM) catalogue (Irwin et al. 1994), recalibrated by E. Flesch (private communication); these magnitudes are flagged with an $O$. The $O$ and Johnson $B$ magnitudes are related by $B-O=-(0.27 \pm 0.06) \times(B-V)($ Evans 1989$)$.

For the SDSS objects we give $V, B-V$ and $U-B$ computed from $u^{\prime}, g^{\prime}$ and $r^{\prime}$ by the following equations (Fukugita et al. 1996):

$$
\begin{aligned}
& V=g^{\prime}-0.53 \times\left(g^{\prime}-r^{\prime}\right) \\
& B-V=0.95 \times\left(g^{\prime}-r^{\prime}\right)+0.22 \\
& U-B=0.72 \times\left(u^{\prime}-g^{\prime}\right)-0.83 .
\end{aligned}
$$

In the other cases, the magnitude given is an estimate as found in the original publications. These magnitudes are generally quite inaccurate and inhomogeneous; they are most often $m_{\mathrm{pg}}$

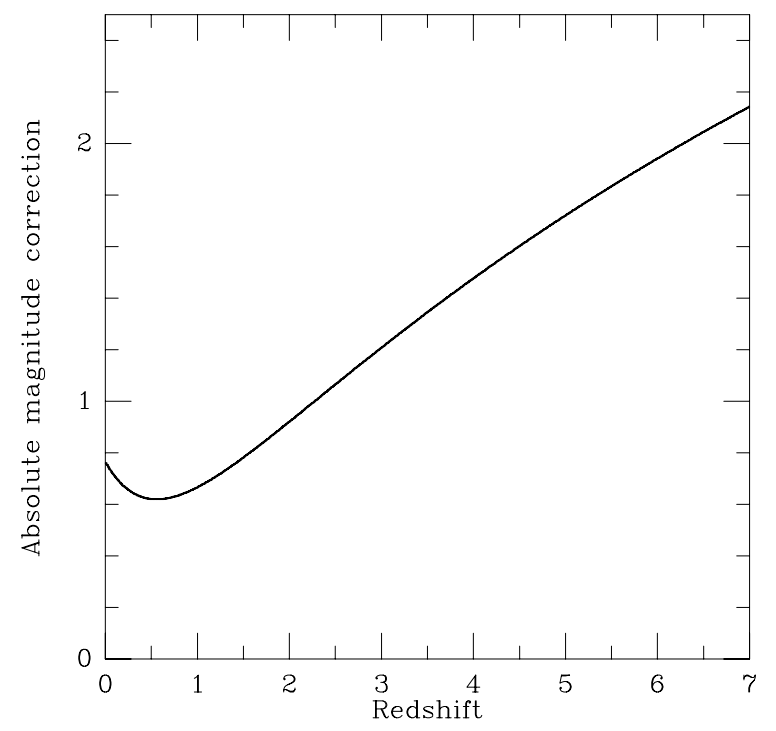

Fig. 2. Correction to be applied to the absolute magnitude if $H_{0}=$ $71 \mathrm{~km} \mathrm{~s}^{-1} \mathrm{Mpc}^{-1}, \Omega_{\mathrm{M}}=0.29$ and $\Omega_{\Lambda}=0.71$ rather than $H_{0}=$ $50 \mathrm{~km} \mathrm{~s}^{-1} \mathrm{Mpc}^{-1}$ and $q_{0}=0$.

or $B$ magnitudes instead of the Johnson $V$ magnitude. Much care should be taken when using them for any purpose. Anyway, even when a photoelectric $V$ magnitude is given, it is not very meaningful as most quasars are variable. On the other hand, the colours of quasars vary little, so the listed colours should be accurate. Again, it should be noted that some of the colours listed are photographic and, therefore, less accurate; moreover, in each catalogue of photoelectric measurements, the faintest objects measured are affected by relatively large errors; this too should not be overlooked. For bright galaxies in Table_AGN, when photoelectric UBV photometry is available, we have chosen the magnitudes and colours measured in the smallest possible diaphragm (preferentially 16 arcsec) as we are interested in the nucleus rather than in the galaxy itself.

7) Column 22. The absolute magnitude $M_{B}$ computed assuming $H_{0}=50 \mathrm{~km} \mathrm{~s}^{-1} \mathrm{Mpc}^{-1}, q_{0}=0$, and an optical spectral index $\alpha$ (defined as $S \propto v^{-\alpha}$ ) equal to 0.3 (Francis et al. 1991), as follows:

$M=m+5-5 \times \log D-k+\Delta m(z)$

where $m$ is the $B$ magnitude, $D=c / H_{0} \times A$, with $A$ the photometric distance (Terrell 1977):

$A=z\left[1+\frac{z\left(1-q_{0}\right)}{\left(1+2 q_{0} z\right)^{0.5}+1+q_{0} z}\right]$

$z$ is the redshift; $k=-2.5 \log (1+z)^{1-\alpha}$ is the $k$ correction, $\Delta m(z)$ is a correction to $k$ taking into account the fact that the spectrum of quasars is not strictly a power law of the form $S \propto v^{-\alpha}$, but is affected by emission lines and by the Ly $\alpha$ forest depleting the continuum to the blue of Ly $\alpha$. Assuming that the spectrum is a power law with $\alpha=0.3$ may not give the best possible estimate of the $k$ correction (Wisotzki 2000). The $R$ magnitudes have been transformed into the $B$ system by using an average $\langle B-R\rangle=0.57$ and the $I$ magnitudes by using $\langle B-I\rangle=1.1$ for low $z$ QSOs. When the reference for the magnitude is Maoz et al. (1993), the magnitude is $V$ and we have used $\langle B-V\rangle=0.40$.

In a more realistic flat cosmology with $H_{0}=$ $71 \mathrm{~km} \mathrm{~s}^{-1} \mathrm{Mpc}^{-1}, \Omega_{\mathrm{M}}=0.29$ and $\Omega_{\Lambda}=0.71$ (see for instance Perlmutter et al. 1999 or Riess et al. 2004), the computed absolute magnitude would be systematically smaller than in the 
Table 2. Gravitationally lensed quasars. Column 1: name, Col. 2: short 1950 position, Col. 3: redshift of the quasar, Col. 4: redshift of the lens, Col. 5: separation in arcsec, Col. 6: references.

\begin{tabular}{|c|c|c|c|c|c|}
\hline$\overline{\text { Name }}$ & Position & $z_{\text {quasar }}$ & $Z_{\text {lens }}$ & $\overline{\operatorname{sep}\left({ }^{\prime \prime}\right)}$ & $\overline{\overline{\text { Ref. }}}$ \\
\hline HE 0047-1756 & $0047-17$ & 1.67 & 0.408 & 1.44 & 78,87 \\
\hline PKS 0132-097 & 0132-09 & 2.216 & 0.764 & 0.7 & $14,18,68$ \\
\hline UM 673 & $0142-10$ & 2.719 & & 2.2 & 60 \\
\hline CTQ 414 & $0156-43$ & 1.29 & & 1.2 & 46 \\
\hline B2 $0218+35$ & $0218+35$ & 0.936 & & 0.33 & 15 \\
\hline HE $0230-2130$ & $0230-21$ & 2.162 & 0.522 & 2.0 & 72,87 \\
\hline Q J0240-343 & $0238-34$ & 1.406 & & 6.1 & 62 \\
\hline SDSS J02465-0825 & $0244-08$ & 1.684 & & 1.04 & 85 \\
\hline PKS 0411+05 & $0411+05$ & 2.639 & 0.958 & 2.2 & 34,64 \\
\hline HE $0435-1223$ & $0435-12$ & 1.689 & 0.456 & 2.6 & 73,80 \\
\hline HE 0512-3329 & $0512-33$ & 1.565 & 0.931 & 0.6 & 12 \\
\hline B $0712+472$ & $0712+47$ & 1.339 & & 1.27 & 8 \\
\hline SDSS J07402+2926 & $0737+29$ & 0.980 & & 2.6 & 84 \\
\hline MG $0751+2716$ & $0748+27$ & 3.200 & 0.350 & & 64 \\
\hline SDSS J08063+2006 & $0803+20$ & 1.540 & & 1.40 & 13 \\
\hline HS $0810+25$ & $0810+25$ & 1.500 & & 0.25 & 55 \\
\hline HS $0818+1227$ & $0818+12$ & 3.115 & & 2.1 & 16 \\
\hline CLASS B0827+525 & $0827+52$ & 2.064 & & 2.8 & 31 \\
\hline APM $08279+5255$ & $0827+52$ & 3.87 & & 0.4 & 35 \\
\hline SDSS $09035+5028$ & $0900+50$ & 3.584 & 0.388 & 2.8 & 28 \\
\hline RX J0911.4+0551 & $0908+06$ & 2.800 & & 0.8 & 1 \\
\hline SBS 0909+532 & $0909+53$ & 1.377 & 0.830 & 1.11 & 30,39 \\
\hline 1WGA J09212+4528 & $0917+45$ & 1.66 & 0.31 & 6.93 & 51 \\
\hline SDSSp J09249+0219 & $0922+02$ & 1.524 & 0.393 & 1.8 & $26,87,88$ \\
\hline FBQS J0951+2635 & $0948+26$ & 1.24 & & 1.1 & 56 \\
\hline BRI 0952-01 & $0952-01$ & 4.43 & & 0.95 & 43 \\
\hline Q $0957+561$ & $0957+56$ & 1.414 & 0.355 & 6.1 & 11,65 \\
\hline SDSS J10014+5027 & $0958+50$ & 1.838 & & 2.86 & 83 \\
\hline FIRST J10044+1229 & $1001+12$ & 2.65 & & 1.54 & 32 \\
\hline RXS J10045+4112 & $1001+41$ & 1.734 & 0.680 & 14.6 & 76 \\
\hline Q 1009-0252 & $1009-02$ & 2.74 & 0.871 & 1.55 & 21,87 \\
\hline $\mathrm{J} 13.03$ & $1015-20$ & 2.55 & & 0.84 & 61 \\
\hline SDSS J10211+4913 & $1018+49$ & 1.720 & & 1.14 & 74 \\
\hline IRAS F10214+4724 & $1021+47$ & 2.286 & & & 57 \\
\hline B $1030+074$ & $1030+07$ & 1.535 & & 1.56 & 8 \\
\hline SDSS J10353+0752 & $1032+08$ & 1.215 & & 2.7 & 84 \\
\hline HE $1104-1805$ & $1104-18$ & 2.303 & 0.729 & 3.0 & 37,70 \\
\hline PG $1115+080$ & $1115+08$ & 1.722 & 0.311 & 2.3 & 63,66 \\
\hline SDSS J11202+6711 & $1117+67$ & 1.494 & & 1.5 & 84 \\
\hline UM 425 & $1120+01$ & 1.465 & & 6.5 & 44 \\
\hline SDSS J11249+5710 & $1122+57$ & 2.311 & & 2.2 & 84 \\
\hline 1RXS J11319-1231 & $1129-12$ & 0.658 & 0.295 & 4.2 & 58 \\
\hline SDSS J11381+6807 & $1135+68$ & 0.769 & & 2.6 & 84 \\
\hline TEX $1152+199$ & $1152+19$ & 1.019 & 0.439 & 1.6 & 52 \\
\hline SDSS J11552+6346 & $1152+64$ & 2.89 & 0.176 & 1.8 & 75 \\
\hline CSO 1270 & $1203+43$ & 1.789 & 0.748 & 2.90 & 83 \\
\hline Q $1208+1011$ & $1208+10$ & 3.803 & & 0.45 & 41 \\
\hline Q $1333+0133$ & $1333+01$ & 1.577 & & 1.6 & 81 \\
\hline SDSS J13531+1138 & $1350+11$ & 1.629 & & 1.41 & 13 \\
\hline 87 GB $1359+1527$ & $1359+15$ & 3.235 & & 1.7 & 52 \\
\hline
\end{tabular}

standard model adopted in the present paper. The correction to add to the absolute magnitude given in this catalogue is given in Fig. 2.

8) The next three columns (23 to 25 ) give the reference for the finding chart, the photometry and the redshift respectively. In many cases, the last reference in Table_AGN is that of the classification of the object (as a Seyfert or otherwise); in these cases the redshift can usually be found in Palumbo et al. (1983).

9) The B1950 position (Cols. 26 to 32).

Since the discovery in 1979 by Walsh et al. of the first gravitationally lensed quasar, Q $0957+561$, a number of such
Table 2. continued.

\begin{tabular}{lllllc}
\hline \hline Name & Position & $z_{\text {quasar }}$ & $z_{\text {lens }}$ & sep(") & Ref. \\
\hline H 1413+117 & $1413+11$ & 2.546 & & 1.4 & 40 \\
HST J14176+5226 & $1415+52$ & 3.4 & & 3.2 & 5 \\
B 1422+231 & $1422+23$ & 3.62 & 0.339 & 1.3 & 53,63 \\
SDSS J15087+3328 & $1506+33$ & 0.878 & 2.9 & 84 \\
SBS 1520+530 & $1520+53$ & 1.855 & 0.717 & 1.6 & 3,4 \\
Q 1600+434 & $1600+43$ & 1.61 & & 1.38 & 27 \\
FIRST J1633+3134 & $1631+31$ & 1.516 & 0.66 & 48 \\
PMN J1632-0033 & $1630-00$ & 3.424 & & 1.46 & 69 \\
Q 1634.9+26.7 & $1634+26$ & 1.961 & & 3.8 & 59 \\
SDSSp J16507+4251 & $1649+42$ & 1.541 & 1.16 & 49 \\
MC 1830-211 & $1830-21$ & 2.507 & 0.885 & 0.60 & 36,38 \\
TEX 1835-345 & $1835-34$ & 2.78 & & 1.0 & 67 \\
MG 2019+1127 & $2016+11$ & 3.273 & & 3.4 & 33 \\
WFI J2026-4536 & $2022-45$ & 2.237 & & 1.4 & 77 \\
WFI J2033-4723 & $2030-47$ & 1.661 & 0.658 & 2.5 & 77,87 \\
87GB 20451+2632 & $2045+26$ & 1.28 & 0.867 & 1.9 & 9 \\
Q 2138-431 & $2138-43$ & 1.641 & & 4.5 & 19 \\
HE 2149-2745 & $2149-27$ & 2.033 & & 1.7 & 71 \\
Q 2237+0305 & $2237+03$ & 1.695 & 0.039 & 1.8 & 24 \\
\hline
\end{tabular}

References:(1) Bade et al. (1997); (2) Brotherton et al. (1999); (3) Burud et al. (2002); (4) Chavushyan et al. (1997); (5) Crampton et al. (1996); (6) Crotts et al. (1994); (7) Djorgovski et al. (1987); (8) Fassnacht \& Cohen (1998); (9) Fassnacht et al. (1999); (10) Faure et al. (2003); (11) Garrett et al. (1992); (12) Gregg et al. (2000); (13) Inada et al. (2006); (14) Gregg et al. (2002); (15) Grundahl \& Hjorth (1995); (16) Hagen \& Reimers (2000); (17) Hagen et al. (1996); (18) Hall et al. (2002); (19) Hawkins et al. (1997); (20) Hewett et al. (1989); (21) Hewett et al. (1994); (22) Hewett et al. (1998); (23) Hewitt et al. (1987); (24) Huchra et al. (1985); (25) Impey et al. (2002); (26) Inada et al. (2003); (27) Jackson et al. (1995); (28) Johnston et al. (2003). (29) Junkkarinen et al. (2001); (30) Kochanek et al. (1997); (31) Koopmans et al. (2000); (32) Lacy et al. (2002); (33) Lawrence et al. (1984); (34) Lawrence et al. (1995); (35) Ledoux et al. (1998); (36) Lidman et al. (1999); (37) Lidman et al. (2000); (38) Lovell et al. (1998); (39) Lubin et al. (2000); (40) Magain et al. (1988); (41) Magain et al. (1992); (42) Mason et al. (2000); (43) McMahon et al. (1992); (44) Meylan \& Djorgovski (1989); (45) Meylan et al. (1990); (46) Morgan et al. (1999); (47) Morgan et al. (2000); (48) Morgan et al. (2001); (49) Morgan et al. (2003); (50) Muñoz et al. (1998); (51) Muñoz et al. (2001); (52) Myers et al. (1999); (53) Patnaïk et al. (1992); (54) Pelló et al. (1996); (55) Reimers et al. (2002); (56) Schechter et al. (1998); (57) Serjeant et al. (1995); (58) Sluse et al. (2003); (59) Steidel \& Sargent, (1991); (60) Surdej et al. (1987); (61) Surdej et al. (1997); (62) Tinney, (1995); (63) Tonry, (1998); (64) Tonry \& Kochanek, (1999); (65) Tytler \& Fan (1992); (66) Weymann et al. (1980); (67) Winn et al. (2000); (68) Winn et al. (2002a); (69) Winn et al. (2002); (70) Wisotzki et al. (1993); (71) Wisotzki et al. (1996); (72) Wisotzki et al. (1999); (73) Wisotzki et al. (2002); (74) Pindor et al. (2006); (75) Pindor et al. (2004); (76) Inada et al. (2003); (77) Morgan et al. (2004); (78) Wisotzki et al. (2004); (79) Green et al. (2004); (80) Morgan et al. (2005); (81) Oguri et al. (2004); (82) Green et al. (2002); (83) Oguri et al. (2005); (84) Hennawi et al. (2006); (85) Inada et al. (2005); (86) Heidt et al. (2003); (87) Ofek et al. (2006); (88) Eigenbrod et al. (2006).

objects (69) and of physical pairs with separation less than $10^{\prime \prime}$ (38) have been found. They are listed in Tables 2 and 3 respectively. Mortlock et al. (1999) have stressed the difficulty sometimes encountered in distinguishing lensed quasars from physical pairs.

Acknowledgements. This research has made use of the APS catalogue of POSS I database which is supported by the National Science Foundation, the National Aeronautics and Space Administration, and the University of Minnesota. We are very grateful to E. Flesch and F. Ochsenbein for checking and improving the catalogue and we thank R. Monella for having brought to our attention a number of errors and omissions in previous editions. 
Table 3. Quasar pairs. Column 1: name, Col. 2: short 1950 position, Col. 3: redshift of the quasar, Col. 4: separation in arcsec, Col. 5: references (see Table 2).

\begin{tabular}{lllcr}
\hline \hline Name & Position & $z$ & sep(") & Ref. \\
\hline LBQS 0015+02 & $0015+02$ & 2.469 & 2.2 & 25 \\
Q 0023+171 & $0023+17$ & 0.945 & 4.8 & 23 \\
SDSS J00480-1051 & $0045-11$ & 1.557 & 3.6 & 84 \\
CT 344 & $0103-27$ & 0.848 & 0.3 & 29 \\
PHL 1222 & $0151+04$ & 1.910 & 3.3 & 45 \\
SDSS J02451-0113 & $0242-01$ & 2.46 & 4.5 & 84 \\
SDSS J02483+0009 & $0245-00$ & 1.645 & 6.9 & 84 \\
CTQ 839 & $0250-33$ & 2.24 & 2.1 & 47 \\
PKS 0537-44 & $0537-44$ & 0.89 & 4.0 & 86 \\
SDSS J07479+4318 & $0744+43$ & 0.50 & 9.2 & 84 \\
SDSS J09591+5449 & $0955+55$ & 1.95 & 3.9 & 84 \\
SDSS J10287+3929 & $1025+39$ & 1.89 & 7.5 & 84 \\
SDSS J10348+0701 & $1032+07$ & 1.25 & 3.1 & 84 \\
2QZ J105644-0059 & $1054-00$ & 2.13 & 7.2 & 84 \\
SDSS J11202+6711 & $1117+67$ & 1.494 & 1.5 & 74 \\
Q 1145-071 & $1145-07$ & 1.34 & 4.2 & 7 \\
HS 1216+5032 & $1216+50$ & 1.450 & 8.9 & 17,79 \\
SDSS J12257+5644 & $1223+57$ & 2.38 & 6.0 & 84 \\
SDSS J12599+1241 & $1257+12$ & 2.19 & 3.6 & 84 \\
SDSS J13033+5100 & $1301+51$ & 1.68 & 3.8 & 84 \\
SDSS J13372+6012 & $1335+60$ & 1.73 & 3.1 & 84 \\
RRS IV 26,27 & $1343+26$ & 2.030 & 9.5 & 6 \\
SDSS J13494+1227 & $1347+12$ & 1.72 & 3.0 & 84 \\
SDSS J14050+4447 & $1403+45$ & 2.23 & 7.4 & 84 \\
SDSS J14098+3919 & $1407+39$ & 2.08 & 6.8 & 84 \\
SDSS J14279-0121 & $1425-01$ & 2.30 & 6.2 & 84 \\
Q 1429-008 & $1429-00$ & 2.076 & 5.1 & 10,20 \\
SDSS J15306+5304 & $1529+53$ & 1.53 & 4.1 & 84 \\
SDSS J16002+0000 & $1557+00$ & 1.010 & 1.8 & 74 \\
RX J16290+3724 & $1627+37$ & 0.923 & 4.3 & 42 \\
Q J1643+31 & $1641+32$ & 0.586 & 2.3 & 2 \\
SDSS J17232+5904 & $1722+59$ & 1.60 & 3.7 & 84 \\
SDSS J21289-0617 & $2126-06$ & 2.07 & 8.3 & 84 \\
Q 2153-2056 & $2153-20$ & 1.845 & 7.8 & 22 \\
SDSS J22144+1326 & $2212+13$ & 2.00 & 5.8 & 84 \\
MGC 2214+3550 & $2212+35$ & 0.877 & 3.0 & 50 \\
SDSS J23365-0107 & $2334-01$ & 1.285 & 1.7 & 14 \\
Q 2345+007 & $2345+00$ & 2.15 & 7.1 & 54,82 \\
\hline & & & & \\
& & & &
\end{tabular}

\section{References}

Abazajian, K., Adelman-McCarthy, J. K., Agüeros, M. A., et al. 2003, AJ, 126, 2081

Abazajian, K., Adelman-McCarthy, J. K., Agüeros, M. A., et al. 2004, AJ, 128, 502

Abazajian, K., Adelman-McCarthy, J. K., Agüeros, M. A., et al. 2005, AJ, 129, 1755

Adelman-McCarthy, J. K., Agüeros, M. A., Allam, S. S., et al. 2006, ApJS, 162, 38

Antonucci, R. R. J., \& Miller, J. S. 1985, ApJ, 297, 621

Aoki, S., Sôma, M., Kinoshita, H., \& Inoue, K. 1983, A\&A, 128, 263

Becker, R. H., White, R. L., \& Helphand, D. J. 1995, ApJ, 450, 559

Bowen, D. V., Osmer, S. J., Blades, J. C., et al. 1994, AJ, 107, 461

Condon, J. J., Cotton, W. D., Greisen, E. W., et al. 1998, AJ, 115, 1693

Cotton, W. D., Condon, J. J., \& Arbizzani, E. 1999, ApJS, 125, 409

Croom, S. M., Smith, R. J., Boyle, B. J., et al. 2001, MNRAS, 322, L29

Croom, S. M., Smith, R. J., Boyle, B. J., et al. 2003, MNRAS, 349, 1397

Crotts, A. P. S., Bechtold, J., Fang, Y., \& Duncan, R. C. 1994, ApJ, 437, L79

De Veny, J. B., Osborn, W. H., \& Janes, K. 1971, PASP, 83, 611

Derry, P. M., O'Brien, P. T., Reeves, J. N., et al. 2003, MNRAS, 342, L53

Eigenbrod, A., Courbin, F., Dye, S., et al. 2006, A\&A, 451, 747

Evans, D. W. 1989, A\&AS, 78, 249

Falco, E. F., Kurtz, M. J., Geller, M. J., et al. 1999, PASP, 111, 438

Fan, X., Strauss, M. A., Schneider, D. P., et al. 1999, AJ, 118, 1

Faure, C., Alloin, D., Gras, S., et al. 2003, A\&A, 405, 415

Fernandez, A., Lortet, M.-C. \& Spite, F. 1983, A\&AS, 52, N 4

Francis, P. J., Hewett, P. C., Foltz, C. B., et al. 1991, ApJ, 373, 465

Fukugita, M., Ichikawa, T., Gunn, J. E., et al. 1996, AJ, 111,1748
Garrett, M. A., Walsh, D., \& Carswell, R. F. 1992, MNRAS, 254, P27

Goodrich, R. W. 1989a, ApJ, 340, 190

Goodrich, R. W. 1989b, ApJ, 342, 224

Goodrich, R. W. 1995, ApJ, 440, 141

Goodrich, R. W., Veilleux, S., \& Hill, G. J. 1994, ApJ, 422, 521

Green, P. J., Kochanek, C., Sieginowska, A., et al. 2002, ApJ, 571,721

Green, P. J., Aldcroft, T. L., Brown, W. R., Kuhn, O., \& Saha, A. 2004, MNRAS, 349,1261

Grundahl, F., \& Hjorth, J. 1995, MNRAS, 275, L67

Hall, P. B., Richards, G. T., York, D. G., et al. 2002, ApJ, 575, L51

Heckman, T. M. 1980, A\&A, 87, 152

Hewitt, A., \& Burbidge, G. 1987, ApJS, 63, 1

Irwin, M., Maddox, S., \& McMahon, R. 1994, Spectrum, 2, 14

Kesteven, M. J. L., \& Bridle, A. H. 1977, J. Roy. Astron. Soc. Canada, 71, 21

Khachikian, E. E., \& Weedman, D. W. 1971, Astrophysics, 7, 231

Khachikian, E. E., \& Weedman, D. W. 1974, ApJ, 192, 581

Kirhakos, S., Sargent, W. L. W., Schneider, D. P., et al. 1994, PASP, 106, 646

Ledoux, C., Theodore, B., Petitjean, P., et al. 1998, A\&A, 339, L77

Lidman, C., Courbin, F., Kneib, J.-P., et al. 2000, A\&A, 364, L62

Lovell, J. E. J., Jauncey, D. L., Reynolds, J. E., et al. 1998, ApJ, 508, L51

Lubin, L. M., Fassnacht, C. D., Reahead, A. C. S., Blandford, R. D., \& Kundic, T. 2000, AJ, 119, 451

McMahon, R., Irwin, M., \& Hazard, C. 1992, Gemini, 36, 1

Magain, P., Surdej, J., Swings, J.-P., Borgeest, U., \& Kayser, R. 1988, Nature, 334,325

Magain, P., Surdej, J., Vanderriest, C., Pirenne, B., \& Hutsemekers, D. 1992, A\&A, 253, L13

Maoz, D., Bahcall, J. N., Schneider, D. P., et al. 1993, ApJ, 409, 28

Meylan, G., Djorgovski, S., Weir, N., \& Shaver, P. 1990, The Messenger, 59, 47

Miller, J. S., \& Goodrich, R. W. 1990, ApJ, 355, 456

Monet, D., Bird, A., Canzian, B., et al. 1996, USNO-A2.0, US Naval Observatory, Washington D.C.

Mortlock, D. J., Webster, R. L., \& Francis, P. J. 1999, MNRAS, 309, 836

Ofek, E. O., Maoz, D., Rix, R.-H., Kochanek, C. S., \& Falco, E. E. 2006, ApJ, 641,70

Oguri, M., Inada, N., Castander, F. J., et al. 2004, PASJ, 56, 399

Osterbrock, D. E. 1977, ApJ, 215, 733

Osterbrock, D. E. 1981, ApJ, 249, 462

Osterbrock, D. E. 1987, Lecture Notes in Physics, 307, 1

Osterbrock, D. E., \& Pogge, R. W. 1985, ApJ, 297, 166

Palumbo, C. G. C., Tanzella-Nitti, G., \& Vettolani, G. 1983, Catalogue of radial velocities of galaxies, Gordon \& Breach

Pelló, R., Miralles, J. M., Le Borgne, J.-F., et al. 1996, A\&A, 314, 73

Pennington, R. L., Humphreys, R. M., Odewahn, S. C., Zumach, W., \& Thurmes, P. M. 1993, PASP, 105, 521

Perlmutter, S., Aldering, G., Knop, R. A., et al. 1999, ApJ, 517, 565

Riess, A. G., Strolger, L.-G., Tonry, J., et al. 2004, ApJ, 607, 665

Schneider, D. P., Bahcall, J. N., Saxe, D. H., et al. 1992, PASP, 104, 678

Serjeant, S., Lacy, M., Rawlings, S., King, L. J., \& Clements, D. L. 1995, MNRAS, 276, L31

Surdej, J., Swings, J.-P., Magain, P., Courvoisier, T. J.-L., \& Borgeest, U. 1987, Nature, 329, 695

Terrell, J. 1977, Am. J. Phys., 45, 869

Tonry, J. L. 1998, AJ, 115, 1

Tran, H. D., Miller, J. S., \& Kay, L. E. 1992a, ApJ, 397, 452

Tran, H. D., Osterbrock, D. E., \& Martel, A. 1992b, AJ, 104, 2072

Treister, E., Castander, F. J., Maccarone, T .J., et al. 2005, ApJ, 621, 104

Véron-Cetty, M.-P., \& Véron, P. 1984, ESO Scientific Rep., No. 1

Véron-Cetty, M.-P., \& Véron, P. 1985, ESO Scientific Rep., No. 4

Véron-Cetty, M.-P., \& Véron, P. 1987, ESO Scientific Rep., No. 5

Véron-Cetty, M.-P., \& Véron, P. 1989, ESO Scientific Rep., No. 7

Véron-Cetty, M.-P., \& Véron, P. 1991, ESO Scientific Rep., No. 10

Véron-Cetty, M.-P., \& Véron, P. 1993, ESO Scientific Rep., No. 13

Véron-Cetty, M.-P., \& Véron, P. 1996a, ESO Scientific Rep., No. 17

Véron-Cetty, M.-P., \& Véron, P. 1996b, A\&AS, 115, 97

Véron-Cetty, M.-P., \& Véron, P. 1998, ESO Scientific Rep., No. 18

Véron-Cetty, M.-P., \& Véron, P. 2000a, ESO Scientific Rep., No. 19

Véron-Cetty, M.-P., \& Véron, P. 2000b, A\&ARv, 10, 81

Véron-Cetty, M.-P., \& Véron, P. 2001, A\&A, 374, 92

Véron-Cetty, M.-P., \& Véron, P. 2003, A\&A, 412, 399

Voges, W., Aschenbach, B., Boller, T., et al. 1999, A\&A, 349, 389

Wadadekar, Y. 2004, A\&A, 416,35

Walsh, D., Carswell, R. F., \& Weymann, R. J. 1979, Nature, 279, 381

White, N. E., Giommi, P., \& Angelini, L. 1994, IAU Circ., 6100

White, R. L., Becker, R. H., Helphand, D. J., \& Gregg, M. 1997, ApJ, 475, 479

Winkler, H. 1992, MNRAS, 257, 677

Winn, J. N., Lovell, J. E. J., Chen, H.-W., et al. 2002a, ApJ, 564, 143

Wisotzki, L. 2000, A\&A, 353, 861 